Draft version March 10, 2021

Preprint typeset using LATEX style emulateapj v. 12/16/11

\title{
TESTING $F(R)$ GRAVITY WITH THE SIMULATED DATA OF GRAVITATIONAL WAVES FROM THE EINSTEIN TELESCOPE
}

\author{
Yu Pan ${ }^{1}$, Yuan He ${ }^{1}$, Jing-Zhao Qi ${ }^{2 *}$, Jin $\mathrm{Li}^{3}$, Shuo CaO ${ }^{4 \dagger}$, Tonghua Liu ${ }^{4}$, And Jun Wang ${ }^{5}$ \\ 1. School of Science, Chongqing University of Posts and Telecommunications, Chongqing 400065, China; \\ 2. Department of Physics, College of Sciences, Northeastern University, Shenyang 110004, China; qijingzhao@mail.neu.edu.cn \\ 3. Department of Physics, Chongqing University, Chongqing 400030, China; \\ 4. Department of Astronomy, Beijing Normal University, Beijing 199875, China; caoshuo@bnu.edu.cn \\ 5. School of Physics and Astronomy, Yunnan University, Kunming 650091, China \\ Draft version March 10, 2021
}

\begin{abstract}
In this paper we analyze the implications of gravitational waves (GWs) as standard sirens on the modified gravity models by using the third-generation gravitational wave detector, i.e., the Einstein Telescope. Two viable models in $f(R)$ theories within the Palatini formalism are considered in our analysis $\left(f_{1}(\mathcal{R})=\mathcal{R}-\frac{\beta}{\mathcal{R}^{n}}\right.$ and $\left.f_{2}(\mathcal{R})=\mathcal{R}+\alpha \ln \mathcal{R}-\beta\right)$, with the combination of simulated GW data and the latest electromagnetic (EM) observational data (including the recently released Pantheon type Ia supernovae sample, the cosmic chronometer data, and baryon acoustic oscillation distance measurements). Our analysis reveals that the standard sirens GWs, which provide an independent and complementary alternative to current experiments, could effectively eliminate the degeneracies among parameters in the two modified gravity models. In addition, we thoroughly investigate the nature of geometrical dark energy in the modified gravity theories with the assistance of $O m(z)$ and statefinder diagnostic analysis. The present analysis makes it clear-cut that the simplest cosmological constant model is still the most preferred by the current data. However, the combination of future naturally improved GW data most recent EM observations will reveal the consistency or acknowledge the tension between the $\Lambda \mathrm{CDM}$ model and modified gravity theories.
\end{abstract}

Keywords: modified gravity, gravitational wave

\section{INTRODUCTION}

The measurement of cosmic acceleration is crucial for our understanding of the nature of the Universe. A number of scenarios have been proposed to explain this remarkable discovery, which fall into two general categories. In the framework of one approach, an exotic component called dark energy is introduced to act the driving force behind the cosmic acceleration (Cao et al. 2011; Cao \& Zhu 2014). Until now, all of the independent astrophysical observations are well consistent with the existence of vacuum energy density or a non-vanishing cosmological constant $\Lambda$ Cao \& Zhu 2012; Cao, Covone \& Zhu 2012; Cao et al. 2012; Pan et al. 2015; Cao et al. 2015a,b, 2017a,b; Li et al. 2017; Risaliti \& Lusso 2018; Liu et al. 2019; Ma et al. 2019). However, such simplest cosmological constant model is still embarrassed by the coincidence problem and fine-tuning problem, which triggered great efforts to understand the evolution of the Universe at early time (Abbott et al. 2017; Qi et al. 2019a; Cao et al. 2019a, 2020; Zheng et al. 2020). On the other hand, such an accelerated expansion is possible with the modification of the gravitational sector in Einstein-Hilbert action (Li et al. 2007; Carroll et al. 2005; Capozziello et al. 2005; De Felice \& Tsujikawa 2010; Sotiriou \& Faraon 2010). Following this direction, a lot of extended theories of gravity have been proposed in the past decades, i.e., Gauss-Bonnet gravity (Noiiri \& Odintsov 2005), $f(T)$ gravity (Bengochea \& Ferraro 2009; Cai et al. 2015; Qi et al. 2016), and $f(R)$ gravity (De Felice \& Tsuiikawa 2010; Sotiriou \& Faraon 2010; Wang et al. 2018). Especially, the $f(R)$ theories of gravity (in which $f(R)$ is a function of the Ricci scalar $R$ ) are widely employed in modern cosmology, benefit from its advantages of describing the large scale structure distribution of the universe (Dos Santos et al. 2016; Voivodic et al. 2017; Wang \& Wang 2013) and explaining the late-time cosmic acceleration. Note that there are mainly two different variational approaches providing various interesting mechanisms, i.e., the metric formalism and the Palatini formalism (Li et al.|2007; Santos et al. 2008; Capozziello \& Francaviglia 2008). In the former formalism, by assuming the connections to be the Christoffel symbols defined in terms of the metric, one can get the generalized field equations by varying the action with respect to the metric, which leads to the fourth-order equations difficult to deal with in practice. Furthermore, it was found in the recent studies that some models within such framework may not only exhibit violent instabilities in the weak gravity regime of matter (Dolgov \& Kawasaki 2003; Nojiri \& Odintsov 2003; Wang et al. 2010), but also fail to produce a standard matter-dominated era followed by an accelerated expansion (Amendola et al. 2006a, b). In this paper, we will mainly investigate the constraints on the Palatini formalism model $\left(R=g^{\mu \nu} R_{\mu \nu}(\Gamma)\right)$, in the framework of which the metric and connection are treated as independent dynamical variables in the action (Vollick 2003; De Felice \& Tsujikawa 2010; Sotiriou \& Faraon 2010). The advantage of the Palatini approach is that the resultant equations are of second order, which are different from the ones derived in the metric approach but more concordant with the field equations in other branches of physics (Vollick 2003; De Felice \& Tsujikawa 2010; 
Sotiriou \& Faraon 2010). Specifically, Palatini gravity have been studied in the context of dark energy models, with the late-time constraints from various astrophysical observations (Bekov et al. 2020; Baghram \& Rahvar 2020; Aoki \& Shimada 2020; Leanizbarrutia et al. 2017; Bohmer et al. 2013; Capozziello et al. 2013; Harko et al. 20212; Gu et al. 2018; Rosa et al. 2020, 2017; Borowiec et al. 2012). Meanwhile, it is interesting to note that some models could exhibit very promising behaviors, i.e., they not only correctly fit different observational data, but also well reproduce the cosmological evolution with early and late-time acceleration (Yang \& Chen 2009; Fav \& Tavakol 2007; Seokcheon 2007). We refer to Sotiriou \& Faraon (2010) for a review on the $f(R)$ gravity.

From observational point of view, it is important to investigate whether these modified gravity theories are indeed compatible with different kinds of observational data. Currently, such analysis has been performed in the EM domain, focusing on different types of standard cosmological probes: type Ia supernovae (SNe Ia), baryon acoustic oscillations (BAO), cosmic microwave background (CMB), and intermediateluminosity radio quasars (ILQSO) Amarzguioui et al. 2006; Fav \& Tavakol 2007; de la Cruz-Dombriz et al. 2016; Song et al. 2007; Xu et al. 2018; Santos et al. 2008). For instance, the first attempt to derive constraints on the $f(R)$ model $f(R)=R-\frac{\beta}{R^{n}}$ was presented in Amarzguioui et al. (2006), which was then extended in (Fay \& Tavakol 2007) by employing the first year SuperNova Legacy Survey (SNLS) data, the baryon acoustic oscillation peak in the SDSS luminous red galaxy sample and the CMB shift parameter. It was found that in subsequent analysis that such $f(R)$ model could produce the sequence of radiation-dominated, matter-dominated, and accelerating periods without the inclusion of dark energy (Santos et al. 2008). However, it is necessary and important to have other complementary cosmological probes in the GW domain, especially the inspiraling and merging compact binaries consisting of neutron stars (NSs) and black holes (BHs) (Schutz 1986). It is well known that the first direct detection of gravitational waves $(\mathrm{GWs})$ by the LIGO/Virgo collaboration (Abbott et al. 2016) has opened the era of GW astronomy. As a promising high-redshift complementary tool to SNe Ia, the greatest advantage of GWs lies in the fact that the distance calibration of such standard siren is independent of any other distance ladders (Taylor et al. 2012). Using the waveform signal to directly measure the luminosity distance $D_{L}$ to the GW sources, the possible cosmological application of these standard sirens has been extensively discussed in the literature (Holz \& Hughes 2005; Dalal et al. 2006; Zhao et al. 2011; Cai \& Yang 2017; Wei 2019; Qi et al. 2019b; Liao 2019; Cao et al. 2019b; Wu et al. 2020; Liu et al. 2020). More interestingly, it was originally proposed (D'Agostino \& Nunes 2019) that standard sirens from binary neutron star mergers could provide strong observational bounds on modified gravity theories (scalar-tensor theories). Specially, the final results showed that the $f(R)$ gravity (i.e., the Hu-Sawicki $f(R)$ gravity model) can be tested at very high precision (95\% confidence level), based on the third generation ground-based GW detector (Einstein telescope).
Such conclusion was further supported by recent measurements of Hubble constant in the context of modified gravity theories ( $f(R)$ and $f(T)$ models), based on the combined geometrical EM data sets obtained in a modelindependent way (D'Agostino \& Nunes 2020). In this paper, we will mainly investigate the constraints on two viable models in $f(R)$ theories within the Palatini formalism $\left(f_{1}(\mathcal{R})=\mathcal{R}-\frac{\beta}{\mathcal{R}^{n}}\right.$ and $\left.f_{2}(\mathcal{R})=\mathcal{R}+\alpha \ln \mathcal{R}-\beta\right)$, focusing on an updated sample of GW events based on the third-generation GW ground-based detector, Einstein Telescope (ET). The latest electromagnetic (EM) observational data, including the recently released Pantheon type Ia supernovae sample, the cosmic chronometers sample and the recent baryon acoustic oscillation measurements are also included in our analysis, in order to achieve a reasonable and compelling constraints on the modified gravity models in both the electromagnetic (EM) and gravitational wave (GW) window.

This paper is organized as follows. In Sec. 2, we review the $f(R)$ theories of gravity, mainly with two variable models within the Palatini formalism. The observational data $(\mathrm{GW}+\mathrm{EM})$ and the corresponding constraint results are introduced in Sec. 3 and 4 , respectively. In Sec. 5, we present some model diagnostics which results in distinction between $f(R)$ models and $\Lambda$ CDM. Finally, we discuss our results and present our main conclusions in Sec. 6.

\section{THE $F(R)$ THEORY IN PALATINI FORMALISM}

We briefly summarize the basic features of $f(R)$ theories of gravity in the Palatini formalism (Sotiriou \& Faraon 2010; Will 1981). The modified Einstein-Hilbert action is given by

$$
S=\frac{1}{16 \pi G} \int d^{4} x \sqrt{-g} f(\mathcal{R})+S_{m},
$$

where $G$ is the gravitational constant, $g$ is the determinant of the metric tensor, and $S_{m}$ is the standard action for the matter fields. In the Palatini formalism, the metric and the connection are completely independent variables. Therefore, the definition of the Ricci scalar can be expressed as $\mathcal{R}=g^{\alpha \beta} \mathcal{R}_{\alpha \beta}\left(\tilde{\Gamma}_{\mu \nu}^{\rho}\right)$, where $\tilde{\Gamma}_{\mu \nu}^{\rho}$ is the affine connection, which is different from the Levi-Civita connection $\Gamma_{\mu \nu}^{\rho}$ in the metric formalism.

Varying the action with respect to the metric and the connection, the field equations can be obtained as

$$
\begin{gathered}
f^{\prime} \mathcal{R}_{(\mu \nu)}-\frac{f}{2} g_{\mu \nu}=8 \pi G T_{\mu \nu}, \\
\tilde{\nabla}_{\alpha}\left[f^{\prime}(\mathcal{R}) \sqrt{-g} g^{\mu \nu}\right]=0,
\end{gathered}
$$

with $f^{\prime} \equiv d f / d \mathcal{R}$ and $f^{\prime \prime} \equiv d^{2} f / d \mathcal{R}^{2}$. Here $T_{\mu \nu} \equiv$ $-\frac{2}{\sqrt{-g}} \frac{\delta S_{m}}{\delta g^{\mu \nu}}$ denotes the energy-momentum tensor of matter, and $\tilde{\nabla}_{\alpha}$ represents the covariant derivative corresponding to the affine connection $\tilde{\Gamma}_{\mu \nu}^{\rho}$. It is obvious that Eq. (2) will reduce to the Einstein's field equations when $f(\mathcal{R})=\mathcal{R}$, while the Einstein's field equations in $\Lambda \mathrm{CDM}$ model can be reproduced when $f(\mathcal{R})=\mathcal{R}-2 \Lambda$.

By introducing a metric conformal $h_{\mu \nu}=f^{\prime}(\mathcal{R}) g_{\mu \nu}$ to Eq. (3), one could derive the definition of the usual 
Levi-Civita connection in terms of the new metric $h_{\mu \nu}$ :

$$
\tilde{\Gamma}_{\mu \nu}^{\lambda}=\frac{h^{\lambda \sigma}}{2}\left(h_{\nu \sigma, \mu}+h_{\mu \sigma, \nu}-h_{\mu \nu, \sigma}\right) .
$$

Then the relation between the generalized affine connection $\tilde{\Gamma}_{\mu \nu}^{\lambda}$ and the metric $g_{\mu \nu}$ can be obtained as

$$
\tilde{\Gamma}_{\mu \nu}^{\lambda}=\Gamma_{\mu \nu}^{\lambda}+\frac{1}{2 f^{\prime}}\left[2 \delta_{(\mu}^{\lambda} \partial_{\nu)} f^{\prime}-g^{\lambda \tau} g_{\mu \nu} \partial_{\tau} f^{\prime}\right] .
$$

Now the relationship between the generalized Ricci tensor $\mathcal{R}_{\mu \nu}$ and the original Ricci tensor $R_{\mu \nu}$ is given by

$$
\mathcal{R}_{\mu \nu}=R_{\mu \nu}-\frac{3}{2} \frac{\nabla_{\mu} f^{\prime} \nabla_{\nu} f^{\prime}}{f^{\prime 2}}+\frac{\nabla_{\mu} \nabla_{\nu} f^{\prime}}{f^{\prime}}+\frac{1}{2} g_{\mu \nu} \frac{\nabla^{\mu} \nabla_{\nu} f^{\prime}}{f^{\prime}},
$$

where

$$
\mathcal{R}_{\mu \nu} \equiv \tilde{\Gamma}_{\mu \nu, \alpha}^{\alpha}-\tilde{\Gamma}_{\mu \alpha, \nu}^{\alpha}+\tilde{\Gamma}_{\alpha \lambda}^{\alpha} \tilde{\Gamma}_{\mu \nu}^{\lambda}-\tilde{\Gamma}_{\mu \lambda}^{\alpha} \tilde{\Gamma}_{\alpha \nu}^{\lambda} .
$$

In the framework of the flat Friedman-RobertsonWalker (FRW) metric

$$
\mathrm{d} s^{2}=-\mathrm{d} t^{2}+a^{2}(t)\left(\mathrm{d} x^{2}+\mathrm{d} y^{2}+\mathrm{d} z^{2}\right)
$$

we take the energy-momentum tensor of matter as a perfect fluid

$$
T_{\mu \nu}=(\rho+p) U_{\mu} U_{\nu}+p g_{\mu \nu},
$$

where $\rho$ is the energy density, $p$ is the pressure and the four-velocity $U_{\mu}$ satisfies $U_{\mu} U^{\mu}=-1$ and $U^{\mu} U_{\mu ; \nu}=0$. The Friedmann equation of the $f(R)$ model can be written as

$$
6\left(H+\frac{1}{2} \frac{\dot{f}^{\prime}}{f^{\prime}}\right)^{2}=\frac{3 f-\mathcal{R} f^{\prime}}{f^{\prime}},
$$

where $H \equiv \dot{a} / a$ is the Hubble parameter, and the overdot denotes the derivative with respect to the cosmic time $t$. Based on the trace of Eq. (2), i.e., $\mathcal{R} f^{\prime}(\mathcal{R})-2 f(\mathcal{R})=\kappa T$ and the conservation equation, i.e., $\dot{\rho}_{\mathrm{m}}+3 H \rho_{\mathrm{m}}=0$, we obtain

$$
\dot{\mathcal{R}}=\frac{3 H \kappa \rho_{\mathrm{m}}}{\mathcal{R} f^{\prime \prime}-f^{\prime}} .
$$

Substituting Eq. (11) into Eq. (10), one can numerically compute the Friedman equation as

$$
H^{2}=\frac{3 f-\mathcal{R} f^{\prime}}{6 f^{\prime} \eta^{2}}
$$

where

$$
\eta=1-\frac{3}{2} \frac{f^{\prime \prime}}{f^{\prime}} \frac{\mathcal{R} f^{\prime}-2 f}{\mathcal{R} f^{\prime \prime}-f^{\prime}} .
$$

According to the definition of the angular diameter distance at redshift $z$, one also has

$$
\begin{aligned}
D_{\mathrm{A}}(z) & =\frac{1}{1+z} \int_{0}^{z} \frac{\mathrm{d} z}{H(z)} \\
& =\frac{1}{3}\left(2 f-\mathcal{R} f^{\prime}\right)^{-\frac{1}{3}} \int_{\mathcal{R}_{0}}^{\mathcal{R}_{z}} \frac{\mathcal{R} f^{\prime \prime}-f^{\prime}}{\left(2 f-\mathcal{R} f^{\prime}\right)^{\frac{2}{3}}} \frac{d \mathcal{R}}{H(\mathcal{R})} .
\end{aligned}
$$

Following the procedure proposed in Fav \& Tavakol (2007), the generalized Friedmann equation can be written in terms of redshift $z=a_{0} / a-1$ and the matter density parameter $\Omega_{m} \equiv \kappa \rho_{m 0} /\left(3 H_{0}^{2}\right)$ as

$$
\frac{H^{2}}{H_{0}^{2}}=\frac{3 \Omega_{m 0}(1+z)^{3}+f / H_{0}^{2}}{6 f^{\prime} \xi^{2}},
$$

where

$$
\xi=1+\frac{9}{2} \frac{f^{\prime \prime}}{f^{\prime}} \frac{H_{0}^{2} \Omega_{m 0}(1+z)^{3}}{R f^{\prime \prime}-f^{\prime}} .
$$

The trace of Eq. (2) leads to

$$
\mathcal{R} f^{\prime}-2 f=-3 H_{0}^{2} \Omega_{m 0}(1+z)^{3},
$$

based on which one may find that Eq. (15) will reduce to General Relativity for the Einstein-Hilbert Lagrangean case $(f(\mathcal{R})=\mathcal{R})$. Moreover, it can be clearly seen that by taking $f(\mathcal{R})=\mathcal{R}-\beta / \mathcal{R}^{n}$, Eq. (17) evaluated at $z=0$ imposes the following relation

$$
\beta=\frac{\mathcal{R}_{0}^{n+1}}{n+2}\left(1-\frac{3 \Omega_{m} H_{0}^{2}}{\mathcal{R}_{0}}\right),
$$

where the present value of the Ricci scalar $\mathcal{R}_{0}$ is determined from the algebraic equation resulting from Eq. (17) and (15) for $z=0$. Therefore, in the framework of $f(R)$ parametrization in the form of $f(\mathcal{R})=\mathcal{R}-\beta / \mathcal{R}^{n}$ and $f(\mathcal{R})=\mathcal{R}+\alpha \ln \mathcal{R}-\beta$ (Fay \& Tavakol 2007), the constraint results on the $f(R)$ model can be shown in the $\left(n, \Omega_{m}\right)$ and $\left(\alpha, \Omega_{m}\right)$ plane.

Now it is necessary to comment on the theoretical applicability of the parameterizations used in this paper. For the former form of $f(\mathcal{R})=\mathcal{R}-\beta / \mathcal{R}^{n}$, Capozziello et al. (2003, 2004) have extensively discussed the possibility of using the models in the metric approach to generate a late-time acceleration of the Universe. Their results showed that for $n>0$ the metric approach was unable to give rise to a standard matter-dominated era followed by a cosmic acceleration Amendola et al. 2006a). However, such theories using the Palatini approach could provide an effective solution to the above difficulties, as was proved in the subsequent analysis of (Fay \& Tavakol 2007). Similarly, for the latter form of $f(\mathcal{R})=\mathcal{R}+\alpha \ln \mathcal{R}-\beta$, the numerical results have also proved the capability of the Palatini approach to produce the sequence of radiation-dominated, matter-dominated, and de Sitter periods (Fay \& Tavakol 2007). Therefore, in this paper we shall focus on a variable $f(R)$ theories recently put forward within the Palatini formalism, the cosmological dynamics of which is still not fully understood up to now. Despite these advantages, some concerns have been expressed on several problems of Palatini $f(R)$ gravity, due to the differential structure of its field equations (Sotiriou \& Faraon 2010), as well as its possible difficulty in passing the solar system tests and providing the correct Newtonian limit (Flanagan 2004a,b). From theoretical point of view, such issue could be potentially fixed by the inclusion of extra terms quadratic in the Ricci and/or Riemann tensor, which is hard to be rigorously accounted in context of cosmological studies like in this paper.

\section{GW SIMULATIONS AND EM OBSERVATIONS}

In this subsection we briefly introduce the method of simulating GW events from Einstein Telescope. Compared with the current advanced ground-based detectors (i.e., the advanced LIGO and Virgo detectors), such 
third-generation GW detector composed of three collocated underground detectors is designed to be ten times more sensitive, especially in the frequency range of $1-10^{4}$ $\mathrm{Hz}$.

We focus on the GW signals generated by the coalescence of binary systems with component masses $m_{1}$ and $m_{2}$, defining the total mass $M=m_{1}+m_{2}$, the symmetric mass ratio $\eta=m_{1} m_{2} / M^{2}$, and the chirp mass $\mathcal{M}_{c}=M \eta^{3 / 5}$. From observational point of view, the observed chirp mass in the observer frame can be written as $\mathcal{M}_{c, \text { obs }}=(1+z) \mathcal{M}_{c \text {,phys. }}$. Note that the GW amplitude depends on the so-called chirp mass and the luminosity distance $D_{L}$, while the former can be measured from the GW signal's phasing. Therefore, the chirping GW signals from inspiraling compact binary stars (NS and $\mathrm{BH}$ ) can provide an absolute measure of the luminosity distance. Following the previous analysis of Zhao et al. (2011); Sathyaprakash et al. (2010), the mass of each black hole and neutron star is assumed to be uniformly distributed in the range $[3,10] M_{\odot}$ and $[1,2] M_{\odot}$. The ratio between NS-NS and $\mathrm{BH}-\mathrm{NS}$ systems is taken to be 0.03 , as is predicted by the Advanced LIGO-Virgo network (Abadie 2010).

GW detectors based on the ET could measure the strain (or the time domain waveform $h(t)$ ) of GWs, the Fourier transform $\mathcal{H}(f)$ of which could be derived by applying the stationary phase approximation

$$
\mathcal{H}(f)=\mathcal{A} f^{-7 / 6} \exp \left[i\left(2 \pi f t_{0}-\pi / 4+2 \psi(f / 2)-\varphi_{(2.0)}\right)\right],
$$

where the Fourier amplitude $\mathcal{A}$ is given by

$$
\begin{aligned}
\mathcal{A}= & \frac{1}{D_{L}} \sqrt{F_{+}^{2}\left(1+\cos ^{2}(\iota)\right)^{2}+4 F_{\times}^{2} \cos ^{2}(\iota)} \\
& \times \sqrt{5 \pi / 96} \pi^{-7 / 6} \mathcal{M}_{c}^{5 / 6},
\end{aligned}
$$

See Zhao et al. (2011) for the definition of the epoch of the merger $t_{0}$, the functions of $\psi$ and $\varphi_{(2.0)}$, the angle of inclination of the binary's orbital angular momentum $\iota$, and the two interferometers' antenna pattern functions $\left(F_{+}, F_{\times}\right)$that depend on the position of GW source, as well as the location and orientation of the detector.

The uncertainty of luminosity distance extracted from the GW signals can be divided into two parts. The first source of uncertainty is quantified by the instrumental uncertainty with the form of

$$
\sigma_{D_{L}}^{\text {inst }} \simeq \sqrt{\left\langle\frac{\partial \mathcal{H}}{\partial D_{L}}, \frac{\partial \mathcal{H}}{\partial D_{L}}\right\rangle^{-1}}
$$

Assuming that this parameter is uncorrelated with any other GW parameters, we add a factor of 2 in the instrumental error to take the effect of inclination into consideration ( $\mathrm{Li}$ 2015)

$$
\sigma_{D_{L}}^{\mathrm{inst}} \simeq \frac{2 D_{L}}{\rho}
$$

Based on the noise power spectral density $S_{h}(f)(\mathrm{PSD})$ of ET given in Zhao et al. (2011), the Signal-to-Noise ratio

\footnotetext{
1 The Einstein Telescope Project, https://www.et-gw.eu/et/
}

(SNR) of the network of three independent interferometers can be calculated as

$$
\rho=\sqrt{\sum_{i=1}^{3}\left\langle\mathcal{H}^{(i)}, \mathcal{H}^{(i)}\right\rangle} .
$$

Here the inner product is defined as

$$
\langle a, b\rangle=4 \int_{f_{\text {lower }}}^{f_{\text {upper }}} \frac{\tilde{a}(f) \tilde{b}^{*}(f)+\tilde{a}^{*}(f) \tilde{b}(f)}{2} \frac{d f}{S_{h}(f)},
$$

where the lower limit in frequency of ET is $f_{\text {lower }}=$ $1 \mathrm{~Hz}$, the upper cutoff frequency is set as $f_{\text {upper }}=$ $2 /\left(6^{3 / 2} 2 \pi M_{\text {obs }}\right)$ and $M_{\text {obs }}=(1+z) M_{\text {phys }}$ is the observed total mass. Note that a GW detection is confirmed if a network SNR is $\rho>8.0$, the SNR threshold currently used by LIGO/Virgo network (Cai \& Yang 2017). The second source of uncertainty is generated by gravitational lensing, which is always modeled as $\sigma_{D_{L}}^{\text {lens }} / D_{L}=0.05 z$ (Sathyaprakash et al. 2010; Li 2015; Cai \& Yang 2017). Thus, the total uncertainty of $D_{L}$ is taken to be

$$
\begin{aligned}
\sigma_{D_{L}} & =\sqrt{\left(\sigma_{D_{L}}^{\text {inst }}\right)^{2}+\left(\sigma_{D_{L}}^{\text {lens }}\right)^{2}} \\
& =\sqrt{\left(\frac{2 D_{L}}{\rho}\right)^{2}+\left(0.05 z D_{L}\right)^{2}} .
\end{aligned}
$$

Thus, the total uncertainty on luminosity distance is

$$
\sigma_{D_{L}}^{2}=\left(\sigma_{D_{L}}^{\text {inst }}\right)^{2}+\left(\sigma_{D_{L}}^{\text {lens }}\right)^{2} .
$$

Focusing on the expected rates of BNS and BHNS detections per year for the ET, only a small fraction of binary mergers may have the observation of detectable EM counterparts like short and intense bursts of $\gamma$ rays (SGRB). In this work we follow the recent analysis of Cai \& Yang (2017) and assume that ET can detect up to 1000 GW events in a 10 year observation (with measurable source redshift). As for the redshift distribution of the observable GW sources, we follow the function taking into account evolution and stellar synthesis (Schneider et al. 2001; Cutler \& Holz 2009; Sathyaprakash et al. 2010), which has been widely applied in gravitational wave cosmology (Qi et al. 2019b). The fiducial cosmological model we choose is the concordant model based on the most recent Plank results (Ade et al. 2016). The GW mock data covering the redshift range $z \sim 5$ are shown in Fig. 11, with the luminosity distance measurements obeying the Gaussian distribution $D_{L}^{\text {mean }}=\mathcal{N}\left(D_{L}^{\text {fid }}, \sigma_{D_{L}}\right)$.

In this analysis, we also include the latest observational data derived in the electromagnetic (EM) window, including the recently released Pantheon type Ia supernovae sample, the baryon acoustic oscillation (BAO) observations, and Hubble parameter measurements obtained through the cosmologicalmodel-independent method using the so-called cosmic chronometers (Jimenez \& Loeb 2002; Jimenez et al. 2003). The type Ia supernovae sample used in our analysis include 1048 SNe Ia observed by the Pan-STARRS1 (PS1) Medium Deep Survey, covering the redshift range of $0.01<z<2.3$ (Scolnic et al. 2018). For the BAO data, we use the six measurement of distance ratio $\frac{d_{A}(z *)}{D_{V}\left(z_{B A O}\right)}$ in the redshift range $0.106 \leq z \leq 0.73$, from 


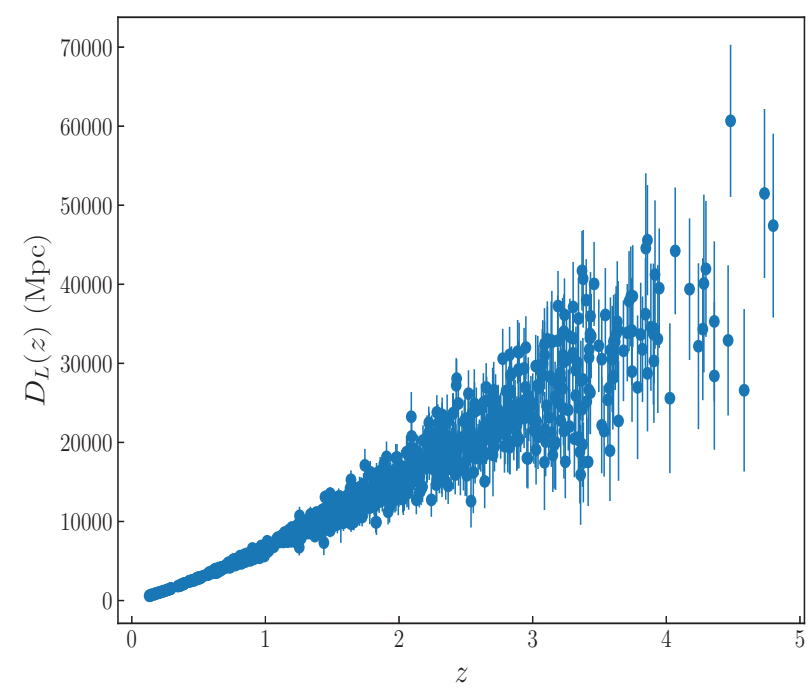

Figure 1. The 1000 simulated GW events from the thirdgeneration gravitational wave detector, i.e., the Einstein Telescope.

the 6-degree Field Galaxy Survey (6dFGS), the WiggleZ galaxy survey, and the Sloan Digital Sky Survey (SDSS-DR7) catalog combined with galaxies from 2dFGRS (Beutler et al. 2011; Ross et al. 2015; Alam et al. 2017; Zhao et al. 2018; Des Bourboux et al. 2017). Here $D_{V}$ is the dilation scale

$$
D_{V}(z)=\left(d_{A}(z)^{2} \frac{z}{H(z)}\right)^{1 / 3}
$$

and $d_{A}$ is the co-moving angular diameter distance (Xu et al. 2018)

$$
d_{A}(z)=\int_{0}^{z} \frac{d z^{\prime}}{H\left(z^{\prime}\right)}
$$

The specific $\mathrm{BAO}$ data and their corresponding fitting procedures are summarized in the source papers (Simon et al. 2005; Stern et al. 2010; Moresco et al. 2012; Moresco 2015; Moresco et al. 2016; Zhang et al. 2014). For the Hubble parameter data, 30 measurements of $H(z)$ covering the redshift range $0.07 \leq z \leq 1.965$ have been obtained, based on the differential age evolution of passively evolving early-type galaxies (Moresco 2015; Moresco et al. 2016). The possible cosmological application of these cosmic chronometers has been extensively discussed in the literature (Ratsimbazafy et al. 2017), such as the reconstruction of cosmological distances (Wu et al. 2020; Zheng et al. 2021) and the spatial curvature of the universe (Liu et al. 2020) in a cosmological model-independent way. In particular, Nunes, Pan \& Saridakis (2017) have recently studied the advantage of utilizing the Hubble parameter data to impose improved constraints on the viable and most used $f(R)$ gravity models, which encourages us to improve and develop it further in this analysis.

\section{CONSTRAINTS AND MODEL DIAGNOSTICS}

4.1. Observational constraints on the $f(R)$ models
In this section we will investigate observational bounds on two $f(R)$ models from a statistical analysis involving three classes of data combinations, i.e., $\mathrm{GW}, \quad \mathrm{EM}(\mathrm{SNe}+\mathrm{BAO}+H(z))$, and $\mathrm{GW}+\mathrm{EM}$ $(\mathrm{GW}+\mathrm{SNe}+\mathrm{BAO}+H(z))$. The Monte Carlo Markov Chain (MCMC) method is applied to perform the minimum likelihood method of $\chi^{2}$ fit (Lewis \& Bridle 2002), with the fitting results shown in Table 1 . The marginalized probability distribution of each parameter $\left(\Omega_{m}, n\right.$ and $H_{0}$ for $f_{1}(\mathcal{R}) ; \Omega_{m}, \alpha$ and $H_{0}$ for $f_{2}(\mathcal{R})$ ) and the marginalized $2 \mathrm{D}$ confidence contours are presented in Fig. 2-3.

Let us start from the first $f(R)$ theory within the Palatini formalism: $f_{1}(\mathcal{R})=\mathcal{R}-\frac{\beta}{\mathcal{R}^{n}}$. For the simulated GW data, the best-fit values of the parameters in the $f_{1}(\mathcal{R})$ model are $\Omega_{m}=0.303_{-0.050}^{+0.073}, H_{0}=67.91 \pm 0.42$ $\mathrm{km} / \mathrm{s} / \mathrm{Mpc}$, and $n=0.01_{-0.24}^{+0.27}$. In order to have a good comparison, the fitting results derived from the $\mathrm{EM}$ observations $(\mathrm{SNe}+\mathrm{BAO}+H(z))$ are also shown in Fig. 2, with the best-fit parameters of $\Omega_{m}=0.303_{-0.028}^{+0.024}$, $H_{0}=64.6 \pm 2.7 \mathrm{~km} / \mathrm{s} / \mathrm{Mpc}$, and $n=0.04_{-0.14}^{+0.11}$. One should note that, although GWs alone seem not able to provide the constraints as good as the current EM observations $(\mathrm{SNe}+\mathrm{BAO}+H(z))$, the degeneracy between different $f(\mathcal{R})$ parameters obtained from the standard sirens is different from the statistical standard probes in the EM window. Such tendency could also be clearly seen from the comparison between the plots obtained with the joint GW+EM analysis, i.e., with the combined standard probe data sets in the GW and EM data sets, the best-fit value for the parameters are $\Omega_{m}=0.285 \pm 0.019$, $H_{0}=67.94 \pm 0.36 \mathrm{~km} / \mathrm{s} / \mathrm{Mpc}$, and $-0.055 \pm 0.086$. Therefore, the shift in the best-fitted parameters (with remarkably reduced allowed region) illustrates how the combination of the future GW observations can be used to improve the model parameters in $f_{1}(\mathcal{R})$ cosmology. This has been noted by the previous analysis studying the constraint ability of the gravitational wave (GW) as the standard siren on the cosmological parameters (Cai \& Yang 2017).

The best-fit values of the parameters along with their $1 \sigma$ uncertainties from three different data combinations are explicitly presented in Table 1 . Note also that in the modified $f_{1}(\mathcal{R})$ gravity, the best-fit value for the matter density parameter and the Hubble parameter, i.e., $\Omega_{m}=0.285 \pm 0.019$ and $H_{0}=67.94 \pm 0.36 \mathrm{~km} / \mathrm{s} / \mathrm{Mpc}$, are well consistent with the current estimates in the framework of $\Lambda \mathrm{CDM}$ cosmology given by recent Planck data release (Aghanim et al. 2018). More importantly, the deviation parameter $n$ from three combinations of data analysis $(\mathrm{EM}+\mathrm{GW}, \mathrm{EM}$ and $\mathrm{GW})$ indicates that $\Lambda \mathrm{CDM}$ model $(n=0)$ is still included within $68.3 \%$ confidence level. However, we can see that the best value of $n=-0.055$ seems to be slightly smaller than 0 , which suggests that there still exists some possibility that $\Lambda \mathrm{CDM}$ may not be the best cosmological model preferred by the current observations. Such conclusion is marginally different from the previous results obtained in Santos et al. (2008); Amarzguioui et al. (2006); Fay \& Tavakol (2007), using the supernova Gold and the SNLS data sets respectively.

Next we turn to study the ability of the standard siren 


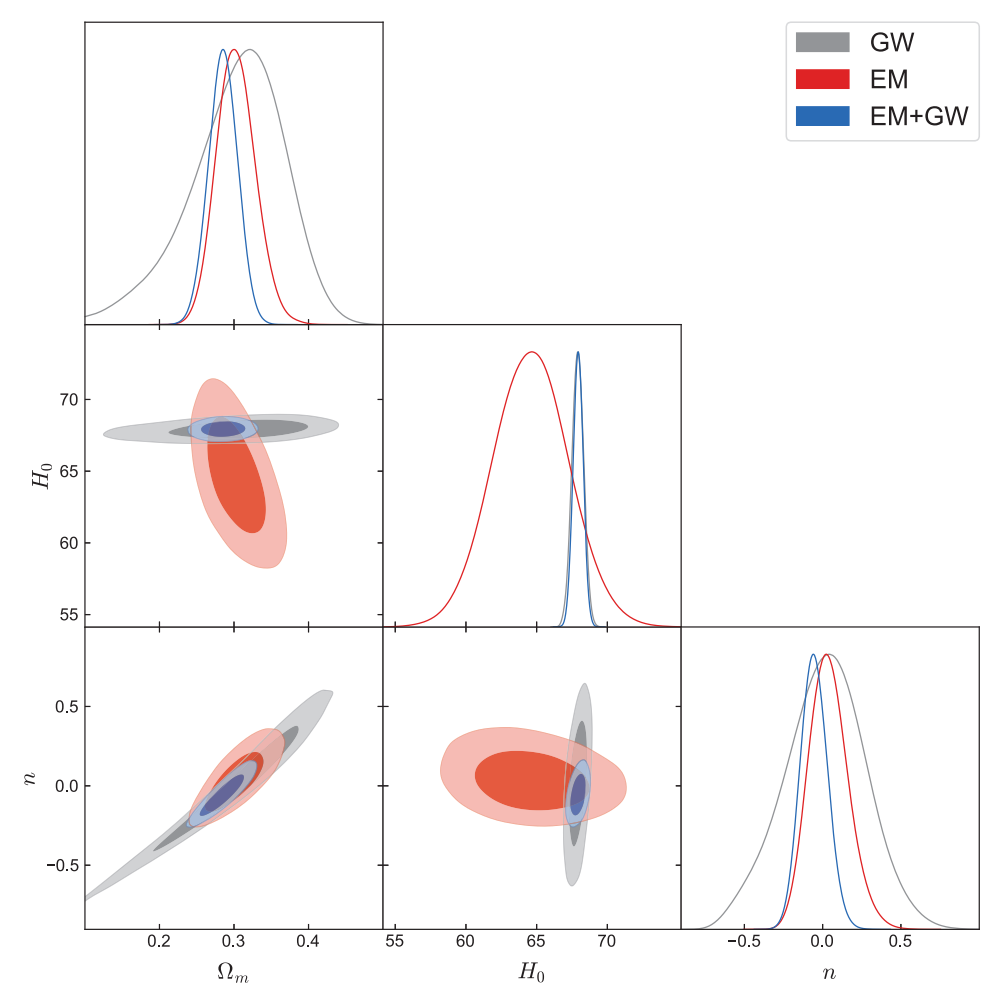

Figure 2. The likelihood distributions of $\Omega_{m}, n$ and $H_{0}$ in the $f_{1}(\mathcal{R})$ model, from the simulated GW data and its combination with the current EM observations.

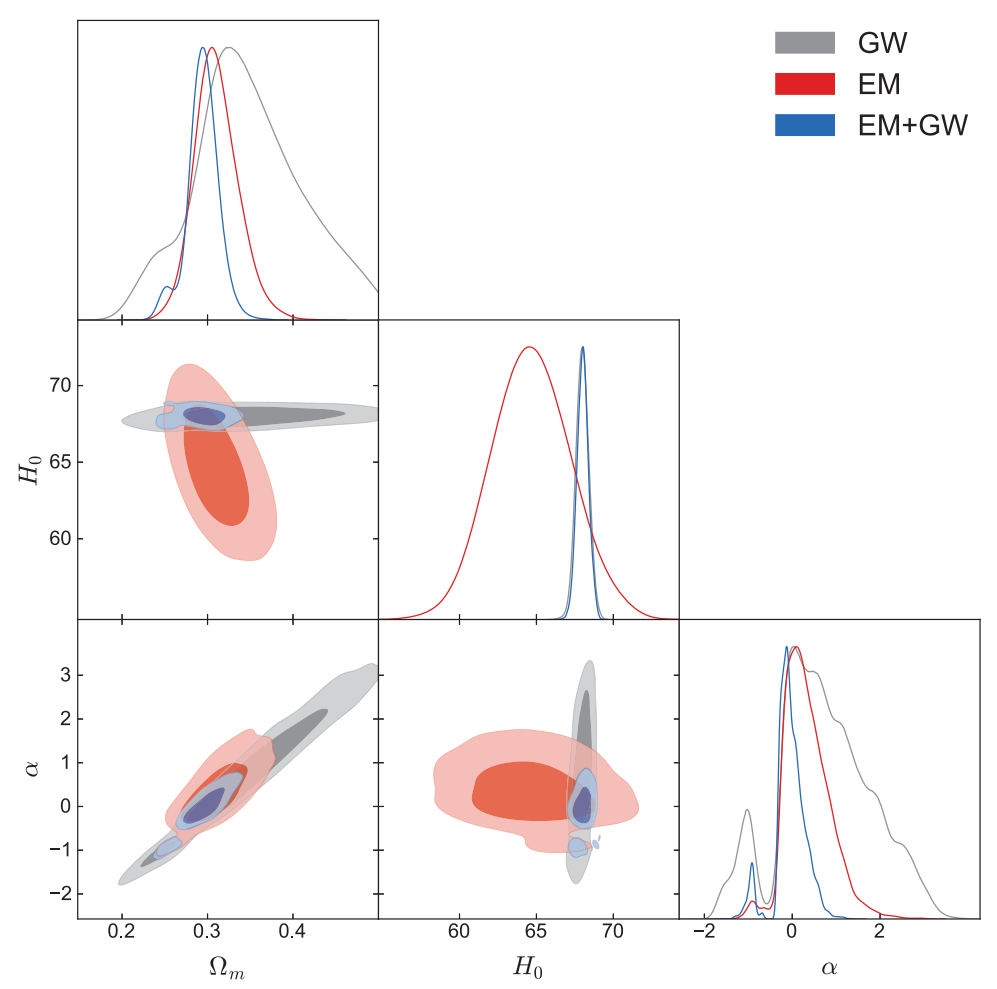

Figure 3. The likelihood distributions of $\Omega_{m}, \alpha$ and $H_{0}$ in the $f_{2}(\mathcal{R})$ model, from the simulated GW data and its combination with the current EM observations. 


\begin{tabular}{|c|c|c|c|}
\hline \hline Data & $\Omega_{m}$ & $H_{0}$ & $n$ \\
\hline $\mathrm{EM}+\mathrm{GW}$ & $0.285 \pm 0.019(1 \sigma) \pm 0.038 \sigma)$ & $67.94 \pm 0.36(1 \sigma) \pm 0.70(2 \sigma)$ & $-0.055 \pm 0.086(1 \sigma)_{-0.16}^{+0.17}(2 \sigma)$ \\
\hline $\mathrm{EM}$ & $0.303_{-0.028}^{+0.024}(1 \sigma)_{-0.049}^{+0.054}(2 \sigma)$ & $64.6 \pm 2.7(1 \sigma)_{-5.2}^{+5.3}(2 \sigma)$ & $0.04_{-0.14}^{+0.11}(1 \sigma)_{-0.24}^{+0.25}(2 \sigma)$ \\
\hline $\mathrm{GW}$ & $0.303_{-0.050}^{+0.073}(1 \sigma)_{-0.13}^{+0.12}(2 \sigma)$ & $67.91 \pm 0.42(1 \sigma) \pm 0.82(2 \sigma)$ & $0.01_{-0.24}^{+0.27}(1 \sigma)_{-0.53}^{+0.50}(2 \sigma)$ \\
\hline \hline Data & $\Omega_{m}$ & $H_{0}$ & $\alpha$ \\
\hline EM+GW & $0.293_{-0.015}^{+0.017}(1 \sigma)_{-0.046}^{+0.035}(2 \sigma)$ & $68.03_{-0.33}^{+0.29}(1 \sigma)_{-0.62}^{+0.69}(2 \sigma)$ & $-0.06 \pm 0.35(1 \sigma)_{-0.97}^{+0.70}(2 \sigma)$ \\
\hline EM & $0.310_{-0.029}^{+0.023}(1 \sigma)_{-0.048}^{+0.056}(2 \sigma)$ & $64.6 \pm 2.6(1 \sigma)_{-5.1}^{+5.2}(2 \sigma)$ & $0.35_{-0.59}^{+0.31}(1 \sigma)_{-0.93}^{+1.2}(2 \sigma)$ \\
\hline GW & $0.347 \pm 0.063(1 \sigma)_{-0.12}^{+0.13}(2 \sigma)$ & $67.99 \pm 0.42(1 \sigma)_{-0.81}^{+0.83}(2 \sigma)$ & $0.7 \pm 1.1(1 \sigma)_{-2.0}^{+2.1 .1}(2 \sigma)$ \\
\hline \hline
\end{tabular}

Table 1

The marginalized $1 \sigma$ uncertainties of the parameters $\Omega_{m}, H_{0}$ and $n(\alpha)$ for the two viable models in $f(R)$ theories.
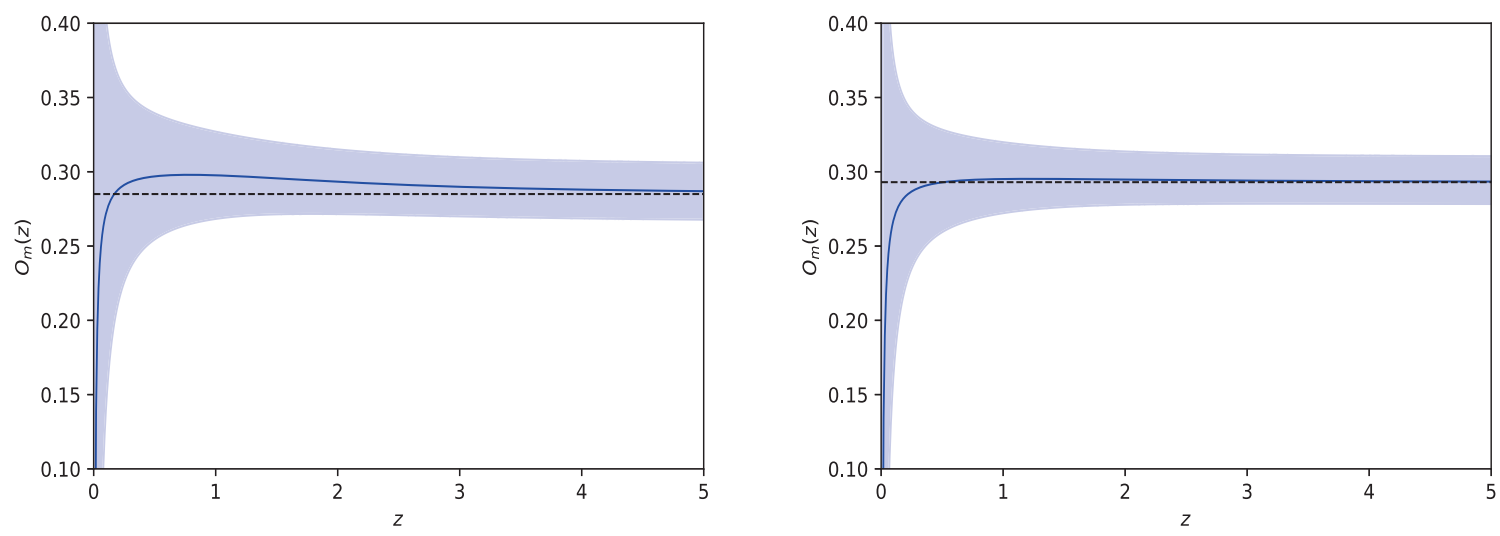

Figure 4. The evolution of $O_{m}(z)$ versus the redshift $z$ for $f_{1}(\mathcal{R})$ and $f_{2}(\mathcal{R})$ model from the simulated EM+GW data, with the $1 \sigma$ uncertainty denoted by blue shades. The black dashed lines represent the standard $\Lambda$ CDM model.
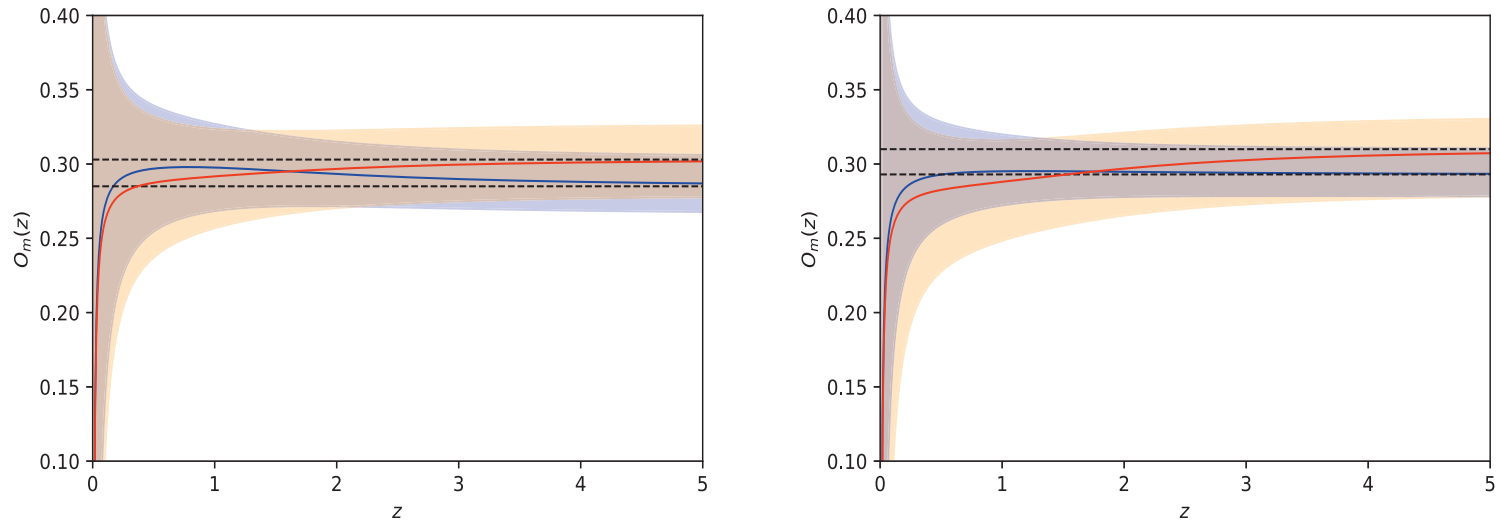

Figure 5. The evolution of $O_{m}(z)$ versus the redshift $z$ for $f_{1}(\mathcal{R})$ and $f_{2}(\mathcal{R})$ model from the EM+GW data and EM data, with the $1 \sigma$ uncertainty denoted by blue shades $(\mathrm{EM}+\mathrm{GW})$, with the $1 \sigma$ uncertainty denoted by oronge shades(EM), The black dashed lines represent the standard $\Lambda$ CDM model. 
to infer the parameters in the $f_{2}(\mathcal{R})$ model: $f_{2}(\mathcal{R})=$ $\mathcal{R}+\alpha \ln \mathcal{R}-\beta$. The best fits takes place at $\Omega_{m}=$ $0.347 \pm 0.063, H_{0}=67.99 \pm 0.42 \mathrm{~km} / \mathrm{s} / \mathrm{Mpc}$ and $\alpha=$ $0.7 \pm 1.1$ with the simulated GW data. The constraining power of the standard sirens in breaking degeneracy between model parameters is slightly existence, as can be seen from the marginalized $1 \sigma$ and $2 \sigma$ contours of each parameter in Fig. 3. By fitting the $f_{2}(\mathcal{R})$ model to the GW+EM observations, we obtain $\Omega_{m}=0.293_{-0.015}^{+0.017}$, $H_{0}=68.03_{-0.33}^{+0.29} \mathrm{~km} / \mathrm{s} / \mathrm{Mpc}$ and $\alpha=-0.06 \pm 0.35$. In Table 1 we also summarize the best-fit values for the three combined data sets, respectively. In broad terms, the estimated values of $\Omega_{m}$ and $H_{0}$ are basically consistent with each other at the $68.3 \%$ confidence level. Moreover, compared with the case in the $f_{1}(\mathcal{R})$ model, the largest difference happens on the constraint of $\alpha$ : the deviation from $\Lambda \mathrm{CDM}$ also tends to be slightly smaller than 0 , although the concordance cosmological scenario is still included within $68.3 \%$ confidence level. Therefore, there still exists some possibility that $\Lambda \mathrm{CDM}$ may not the best cosmological model preferred by the current and future high-redshift GW+EM observations. The constraining ability of standard siren data can be quantified by comparing the results at $1 \sigma \mathrm{C}$. L. based on GW+EM analysis, i.e., we obtain the error bar smaller than that from EM data alone, when the $f_{2}(\mathcal{R})$ model is considered.

\subsection{Model diagnostics}

Now two diagnostics analysis (the $\operatorname{Om}(z)$ and the statefinder diagnostics) will be performed in this section, acting as alternative probes capable of discriminating between $f(R)$ cosmology and $\Lambda$ CDM model.

On the one hand, the expansion rates at different redshifts, or the Hubble parameters $H(z)$, has opened a new chapter of using the so-called $\operatorname{Om}(z)$ diagnostics to discriminate different cosmological models as well as $\Lambda$ CDM model (Sahni et al. 2008; Ding et al. 2015; Qi et al. 2018; Zheng et al. 2016, 2019). In this method, only a single time derivative of $a(t)$ is used and a new diagnostics is defined as

$$
O m(z)=\frac{E^{2}(z)-1}{(1+z)^{3}-1}
$$

where $E(z)=\frac{H(z)}{H_{0}}$ represents the dimensionless expansion rate. It is obvious that in the flat $\Lambda \mathrm{CDM}$ model, the $\operatorname{Om}(z)$ evaluated at any redshift is always equal to the present mass density parameter $\Omega_{m}$. Therefore, such diagnostic can be used as a cosmological probe to directly illustrate the difference between $\Lambda \mathrm{CDM}$ and other cosmological models.

Based on the best-fit model parameters and their $1 \sigma$ uncertainties derived from the simulated $\mathrm{EM}+\mathrm{GW}$ data, we have different values of $O m(z)$ as the function of redshift for the $\Lambda \mathrm{CDM}$ and two $f(R)$ models, which are presented in Fig. 4. It is obvious that the $O_{m}(z)$ curve of the $f_{1}(\mathcal{R})$ model will coincide with $\Lambda$ CDM at high redshifts $(z>4)$, which indicates that the $O_{m}(z)$ for the $f_{1}(\mathcal{R})$ model cannot be distinguished $\Lambda$ CDM in the early universe. However, the $f_{1}(\mathcal{R})$ cosmology begins to deviate from $\Lambda C D M$ in the redshift range of $z<4$, while the deviation between $f_{2}(\mathcal{R})$ model and $\Lambda$ CDM takes place at the redshift of $z>1.5$. In Fig. 5 we also compare the $O_{m}(z)$ function based on the fits from $\mathrm{EM}+\mathrm{GW}$ and
EM data. One may clearly see that in the framework of two $f(R)$ models, the combination of future naturally improved GW data most recent EM observations will reveal the consistency or acknowledge the tension between the $\Lambda$ CDM model and modified gravity theories.

On the other hand, in order to more effectively differentiate and compare between different cosmological models, we turn to a discussion on the so-called statefinder diagnostic. For a specific cosmological model, the Hubble parameter $H$ and the deceleration parameter $q$ are respectively related as

$$
q(z)=-\frac{\ddot{a}}{a H^{2}}=\frac{E^{\prime}(z)}{E(z)}(1+z)-1
$$

In the analysis of statefinde diagnostic Sahni et al. 2008), the new dimensionless parameters $r, s$ are constructed from the scale factor $a(t)$ and its time derivatives similar to the geometrical parameters $H(z)$ and $q(z)$ :

$$
\begin{aligned}
& r(z)=q(z)(1+2 q(z))+q^{\prime}(z)(1+z) \\
& s(z)=\frac{1}{3}(r(z)-1) /(q(z)-1 / 2)
\end{aligned}
$$

where $q^{\prime}(z) \equiv \frac{d q(z)}{d z}$. Any obtained model can be compared with the standard cosmological constant model, from which one may see how a model approaches or deviates from $\Lambda \mathrm{CDM}$ in the $s-r$ plane and $q-r$ plane. The evolution of starefinder pair $(s, r)$ in the two $f(R)$ models is shown in Fig. 7. On the one hand, it is obvious that the statefinder pair exhibits the similar behaviors in the framework of two $f(R)$ models, i.e., originating from the same point of $(r, q)=(1,0.5)$, evolving along different trajectory, and finally converging on the same point of $(1,-1)$. On the other hand, compared with the deceleration parameter $q$, the parameter $r$ exhibiting apparent fluctuations is more effective in discriminating different cosmological models. The trajectories of the two $f(R)$ models in the $r-q$ plane are also presented in Fig. 6 in which the point $(r, s)=(1,0)$ corresponding to the $\Lambda \mathrm{CDM}$ universe is also added for comparison. It is noteworthy that, although the corresponding value for the two $f(R)$ models significantly deviates from $\Lambda$ CDM at the present epoch, both of two modified gravity models discussed in this analysis is resembling to the standard cosmological model in the future and ultimately freezing to it. Such a tendency is more obvious when the $1 \sigma$ uncertainties of the $f(R)$ parameters are taken into consideration.

\section{CONCLUSIONS}

In this paper we have investigated the constraint ability of GW events on the modified gravity models using the simulated data from the third-generation gravitational wave detector, the Einstein Telescope. We focus on two $f(R)$ models which introduces a perturbation of the Ricci scalar $R$ in the Einstein-Hilbert action, which can be considered as an important candidate models alternative to dark energy. More specifically, in the framework of two viable models in $f(R)$ theories within the Palatini formalism $\left(f_{1}(\mathcal{R})=\mathcal{R}-\frac{\beta}{\mathcal{R}^{n}}\right.$ and $f_{2}(\mathcal{R})=\mathcal{R}+\alpha \ln \mathcal{R}-\beta$ ), our results show that the sensitivity achieved by the ET detector or a similar third generation interferometer is enough to improve the current estimates on the free parameter within $f(R)$ gravity. 

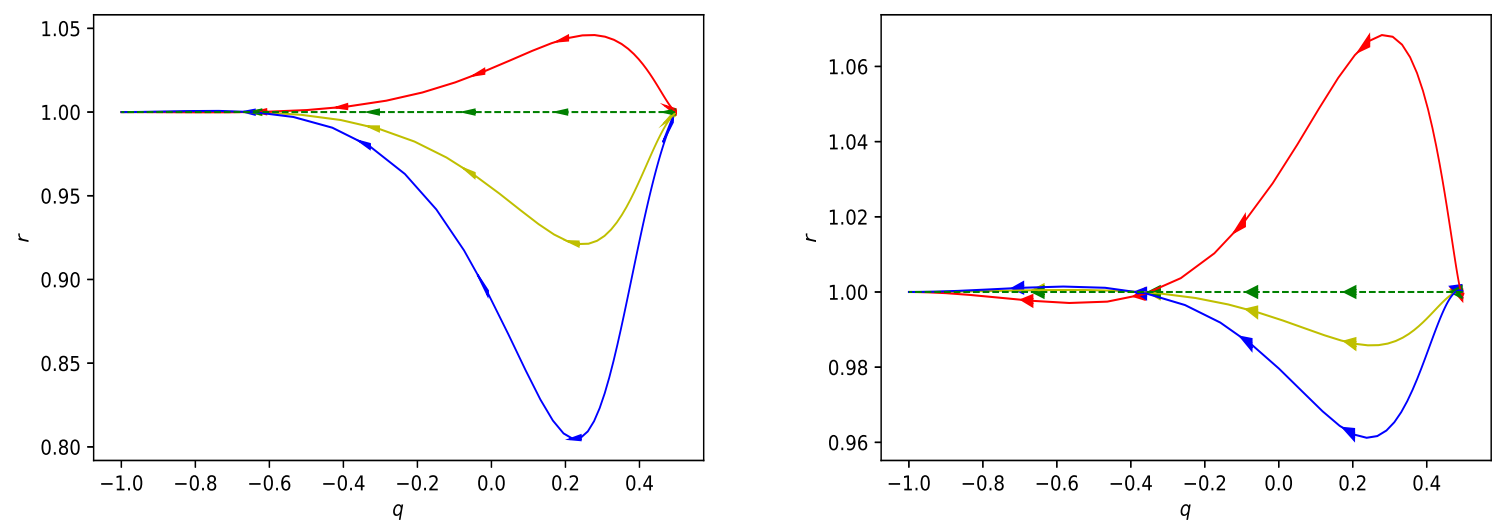

Figure 6. The plot shows the behavior of the $f_{1}(\mathcal{R})$ model and $f_{2}(\mathcal{R})$ model in the $(r, q)$ plane. The red and blue lines represent the evolution of $(r, q)$ within the $1 \sigma$ error range.

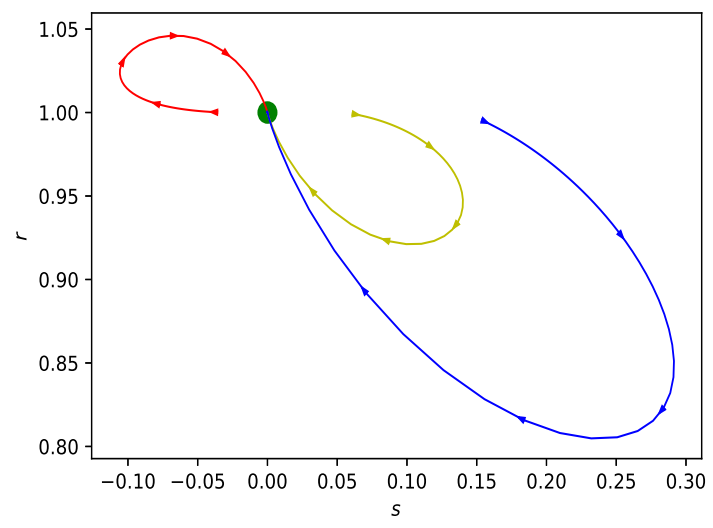

Figure 7. The same as Fig. 6, but in the $(r, s)$ plane.

In practise more GW events may be needed in order to achieve the accuracy of EM data. More importantly, the standard sirens GWs could effectively eliminate the degeneracies among parameters in the two modified gravity models, in joint analysis with the latest electromagnetic (EM) observational data (including the recently released Pantheon type Ia supernovae sample, Hubble parameter data from cosmic chronometers, and baryon acoustic oscillation distance measurements).

In addition, we thoroughly investigate the nature of geometrical dark energy in the modified gravity theories with the assistance of $\mathrm{Om}(z)$ and statefinder diagnostic analysis. The present analysis makes it clear that the simplest cosmological constant model is still the most preferred by the current data. However, the combination of future naturally improved GW data most recent EM observations will reveal the consistency or acknowledge the tension between the $\Lambda \mathrm{CDM}$ model and modified gravity theories. Therefore, in the case of detections with the ET, our forecast analysis indicates that it is possible to provide an independent and complementary alternative to current experiments, once that these events begin to be detected. One should note that, dedicated observations of the sky position of each host galaxy (i.e., with negligible measurement error) would be necessary, in order to obtain significant improvements in the distance,

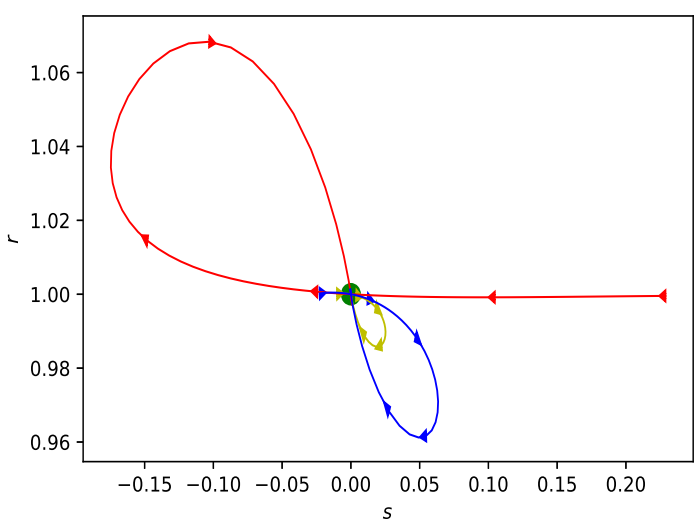

and hence in the constraints on modified gravity theories. Although the GW distance posterior changes slowly over the sky and therefore is not sensitive to the precise location of the counterpart, obtaining such measurements for a sample of different types of GW events would require substantial follow-up efforts (Zhang et al. 2020).

\section{ACKNOWLEDGMENTS}

We thank Prof. Z.-H. Zhu for useful discussions. This work was supported by National Key R\&D Program of China No. 2017YFA0402600; the National Natural Science Foundation of China under Grants Nos. 12021003, 11690023, and 11633001; Chongqing Municipal Science and Technology Commission Fund (cstc2018jcyjAX0192); Beijing Talents Fund of Organization Department of Beijing Municipal Committee of the CPC; the Strategic Priority Research Program of the Chinese Academy of Sciences, Grant No. XDB23000000; the Interdiscipline Research Funds of Beijing Normal University; and the Opening Project of Key Laboratory of Computational Astrophysics, National Astronomical Observatories, Chinese Academy of Sciences. J. Wang was supported by the National Natural Science Foundation of China under Grant No.11647079, Science and Technology Department of Yunnan Province - Yunnan University Joint Funding (2019FY003005), Donglu Youth Teacher Plan of Yunnan University and the Key 
Laboratory of Astroparticle Physics of Yunnan Province.

\section{REFERENCES}

Abadie, J., et al. 2010, Nucl. Instrum. Methods Phys. Res., 624, 223

Abbott, B. P., et al. 2016, PRL, 116, 061102

Abbott, B. P., et al. 2017, Nature, 551, 85

Ade, P. A. R., Aghanim, N., et al. 2016, A\&A, 594, A13

Aghanim, N., Akrami, Y., Ashdown, M., et al. 2018, arXiv: 1807.06209

Alam, S., Ata, M., Bailey, S., et al. 2017, MNRAS, 470, 2617

Amendola, L., et al. 2006a, arXiv:0603703

Amendola, L., et al. 2006b, arXiv:0612180

Amarzguioui, M., et al. 2006, A\&A, 454, 707

Aoki, K. \& Shimada, K. 2020, PRD, 98, 044038

Baghram, S. \& Rahvar, S. 2020, PRD, 80, 124049

Bekov, S., Myrzakulov, K.,Myrzakulov, R. \& Gomez, D. S. C. 2020, arXiv:2010.12360 v2 [gr-qc]

Bengochea, G. R. \& Ferraro, R. 2009, PRD, 79, 124019

Beutler, F., Blake, C., Colless, M., et al. 2011, MNRAS, 416, 3017

Bohmer, C. G., Lobo, F. S. N. \& Tamanini, N. 2013, PRD, 88, 104019

Borowiec, A., Kamionka, M., Kurek, A. \& Szydlowski, M. 2012, JCAP, 2, 027

Cai, Y.-F., et al. 2015, arXiv:1511.07586

Cai, R.-G. \& Yang, T. 2017, PRD, 95, 044024

Cao, S., Zhu, Z.-H., \& Zhao, R. 2011, PRD, 84, 023005

Cao, S., \& Zhu, Z.-H. 2012, A\&A, 538, A43

Cao, S., Covone, G., \& Zhu, Z.-H. 2012, ApJ, 755, 31

Cao, S., Pan, Y., Biesiada, M., Godlowski, W., \& Zhu Z.-H. 2012, JCAP, 03, 016

Cao, S., \& Zhu, Z.-H. 2014, PRD, 90, 083006

Cao, S., et al. 2015a, AJ, 149, 3

Cao, S., Biesiada, M., Gavazzi, R., Piórkowska, A., \& Zhu, Z.-H. 2015b, ApJ, 806, 185

Cao, S., et al. 2017, JCAP, 02, 012

Cao, S., et al. 2017, A\&A, 606, A15

Cao, S., Qi, J. Z., Biesiada, M., et al. 2019a, PDU, 24, 100274

Cao, S., et al. 2019b, NatSR, 9, 11608

Cao, S., Qi, J. Z., Biesiada, M., Liu, T. H., Zhu, Z.-H. 2020, ApJL, 888, L25

Capozziello, S., et al. 2003, PRD, 12, 1969

Capozziello, S., et al. 2004, PRD, 70, 043528

Capozziello, S., Harko, T., Koivisto, T. S., Lobo, F. S. N. \& Olmo, G. J. 2013, JCAP, 4, 011

Capozziello, V. F. S., et al. 2005, PRD, 71, 043503

Capozziello, S. \& Francaviglia, M. 2008, Gen. Relativ. Gravit, 40 357

Carroll, S. M., et al. 2005, PRD, 71, 063513

Chen, L., Huang, Q.-G., \& Wang, K. 2019, JCAP, 02, 028

Cutler, C. \& Holz, D. E. 2009, PRD, 80, 104009

D'Agostino, R. \& Nunes, R. C. 2019, PRD, 100, 044041

D'Agostino, R. \& Nunes, R. C. 2020, PRD, 101, 103505

Dalal, N., et al. 2006, PRD, 74, 063006

De Felice, A. \& Tsujikawa, S. 2010, Living Rev. Relativ, 13, 3

de la Cruz-Dombriz, A., et al. 2016, PRD, 93, 084016

Des Bourboux, H. D. M., Le Goffff, J.-M., Blomqvist, M., et al. 2017, A\&A, 608, A130

Ding, X., Biesiada, M., Cao, S., Li, Z., \& Zhu, Z.-H. 2015, ApJL, 803, L22

Dolgov, A. D. \& Kawasaki, M. 2003, PLB, 573, 1

Dos Santos, M. V., et al. 2016, A\&A, 587, A132

Fay, S. \& Tavakol, R. 2007, PRD, 75, 063509

Flanagan, E. E. 2004a, PRL, 92, 071101

Flanagan, E. E. 2004b, CQG, 21, 417

Gu, B. M., Liu, Y. X. \& Zhong, Y. 2018, PRD, 98, 024027

Jimenez, R. \& Loeb, A. 2002, ApJ, 573, 37

Jimenez, R., Verde, L., Treu, T. \& Stern, D. 2003, ApJ, 593, 622

Harko, T., Koivisto, T. S., Lobo, F. S. N. \& Olmo, G. J. 2012,

PRD, 85, 084016
Holz, D. E. \& Hughes, S. A. 2005, ApJ, 629, 15

Leanizbarrutia, I., Lobo, F. S. N. \& Saez-Gomez, D. 2017, PRD, 95, 084046

Lewis, A. \& Bridle, S. 2002, PRD, 66, 103511

Li, B., Chan, K. C., \& Chu, M.-C. 2007, PRD, 76, 024002

Li, T. G. F., Extracting Physics from Gravitational Waves (Springer Theses, New York (2015)

Li, X. L., et al. 2017, EPJC, 77, 677

Liao, K. 2019, ApJ, 885,70

Liu, T. H., et al. 2019, ApJ, 886, 94

Liu, T. H., et al. 2020, ApJ, 899, 71

Liu, Y. T., et al. 2020, ApJ, 901, 129

Ma, Y. B., et al. 2019, EPJC, 79, 121

Moresco, M. 2015, MNRAS, 450, L16

Moresco, M., et al. 2016, JCAP, 5, 014

Moresco, M., Verde, L., Pozzetti, L., Jimenez, R. \& Cimatti, A. 2012, JCAP, 7, 053

Nojiri, S. \& Odintsov, S. D. 2003, PRD, 68, 123512

Nojiri, S. \& Odintsov, S. D. 2005, PLB, 631, 1

Nunes, R. C., Pan, S., \& Saridakis, E. N. 2017, JCAP, 01, 005

Pan, Y., et al. 2015, ApJ, 808, 78

Qi, J.-Z., et al. 2016, 16, 002

Qi, J.-Z., et al. 2018, RAA, 18, 66

Qi, J.-Z., et al. 2019a, MNRAS, 483, 1

Qi, J.-Z., et al. 2019b, PRD, 99, 6

Ratsimbazafy, A. L., et al. 2017, MNRAS, 467, 3239

Risaliti, G., \& Lusso, E. 2019, Nature Astronomy, 3, 272

Ross, A. J., Samushia, L., Howlett, C., et al. 2015, MNRAS, 449, 835

Rosa, J. L., Carloni, S. \& Lemos, J. P. S. 2020, PRD, 101, 104056

Rosa, J. L., Carloni, S., Lemos, J. P. S. \& Lobo, F. S. N.. 2017, PRD, 95, 124035

Sahni, V., Shafieloo, A., \& Starobinsky, A. A. 2008, PRD, 78, 103502

Santos, J., et al. 2008, PLB, 669, 14

Sathyaprakash, B. S., et al. 2010, CQG, 27, 215006

Schneider, R., et al. 2001, MNRAS, 324, 797

Schutz, B. F. 1986, Nature, 323, 310

Scolnic, D. M., Jones, D. O., Rest, A., et al. 2018, ApJ, 859, 101

Seokcheon L. 2007, arXiv:0710.2395

Simon, J., Verde, L. \& Jimenez, R. 2005, PRD, 71, 123001

Song, Y.-S., Peiris, H., \& Hu, W. 2007, PRD, 76, 063517

Sotiriou, T. P. \& Faraoni, V. 2010, Reviews of Modern Physics, 82,451

Stern, D., Jimenez, R., Verde, L., Kamionkowski, M. \& Stanford, S. A. 2010, JCAP, 2, 008

Taylor, S. R., et al. 2012, PRD, 85, 023535

Voivodic, R., et al. 2017, PRD, 95, 024018

Vollick, D. N. 2003, PRD, 68, 063510

Wang, J., et al. 2010, PLB, 689, 133

Wang, J., et al. 2018, PDU, 19, 60

Wang, J. \& Wang, H. 2013, PLB, 724, 5

Wei, J.-J. 2019, ApJ, 876, 1

Will, C. M. 1981, Theory and Experiment in Gravitational Physics (Cambridge University Press, New York)

Wu, Y., et al. 2020, ApJ, 888, 113

$\mathrm{Xu}, \mathrm{T}$., et al. 2018, JCAP, 06, 042

Yang, X. J. \& Chen, D. M. 2009, MNRAS, 394, 3

Zhang, C. et al. 2014, RAA, 14, 1221

Zhang, S. X., et al. 2020, IJMPD, 2020, 2050105

Zhao, W., et al. 2011, PRD, 83, 023005

Zhao, G.-B., Wang, Y., Saito, S., et al. 2018, MNRAS, 482, 3

Zheng, X.-G., Ding, X., Biesiada, M., Cao, S., \& Zhu, Z.-H. 2016, ApJ, 825, 17

Zheng, X.-G., et al. 2019, EPJC, 79, 637

Zheng, X.-G., et al. 2020, ApJ, 892, 103

Zheng, X.-G., et al. 2021, SCMPA, in press arXiv:2012.14607 